\title{
A comparison of conditional random fields and structured support vector machines for chemical entity recognition in biomedical literature
}

Buzhou Tang ${ }^{1,2}$, Yudong Feng ${ }^{3}$, Xiaolong Wang ${ }^{1 *}$, Yonghui Wu ${ }^{2}$, Yaoyun Zhang ${ }^{2}$, Min Jiang ${ }^{2}$, Jingqi Wang ${ }^{2}$, Hua $\mathrm{Xu}^{2^{*}}$

\begin{abstract}
Background: Chemical compounds and drugs (together called chemical entities) embedded in scientific articles are crucial for many information extraction tasks in the biomedical domain. However, only a very limited number of chemical entity recognition systems are publically available, probably due to the lack of large manually annotated corpora. To accelerate the development of chemical entity recognition systems, the Spanish National Cancer Research Center (CNIO) and The University of Navarra organized a challenge on Chemical and Drug Named Entity Recognition (CHEMDNER). The CHEMDNER challenge contains two individual subtasks: 1) Chemical Entity Mention recognition (CEM); and 2) Chemical Document Indexing (CDI). Our study proposes machine learningbased systems for the CEM task.

Methods: The 2013 CHEMDNER challenge organizers provided a manually annotated 10,000 UTF8-encoded PubMed abstracts according to a predefined annotation guideline: a training set of 3,500 abstracts, a development set of 3,500 abstracts and a test set of 3,000 abstracts. We developed machine learning-based systems, based on conditional random fields (CRF) and structured support vector machines (SSVM) respectively, for the CEM task for this data set. The effects of three types of word representation (WR) features, generated by Brown clustering, random indexing and skip-gram, on both two machine learning-based systems were also investigated. The performance of our system was evaluated on the test set using scripts provided by the CHEMDNER challenge organizers. Primary evaluation measures were micro Precision, Recall, and F-measure.
\end{abstract}

Results: Our best system was among the top ranked systems with an official micro F-measure of $85.05 \%$. Fixing a bug caused by inconsistent features marginally improved the performance (micro F-measure of $85.20 \%$ ) of the system.

Conclusions: The SSVM-based CEM systems outperformed the CRF-based CEM systems when using the same features. Each type of the WR feature was beneficial to the CEM task. Both the CRF-based and SSVM-based systems using the all three types of WR features showed better performance than the systems using only one type of the WR feature.

\section{Background}

Chemical compounds and drugs (together called chemical entities) embedded in scientific articles are crucial for many information extraction tasks in the biomedical

\footnotetext{
*Correspondence: wangxl@insun.hit.edu.cn; hua.xu@uth.tmc.edu ${ }^{1}$ Department of Computer Science, Harbin Institute of Technology Shenzhen Guraduate, Shenzhen, Guangdong, China

${ }^{2}$ School of Biomedical Informatics, The University of Texas Health Science Center at Houston, Houston, Texas, USA

Full list of author information is available at the end of the article
}

domain, such as detection of drug-protein interactions and adverse drug reactions [1]. Recognizing chemical entities from biomedical literature is a typical named entity recognition (NER) task. Compared with other NER tasks, such as NER in newswire domain [2], biomedical NER in biomedical domain $[3,4]$ and clinical NER in clinical domain [5], there are many unique challenges in chemical entity recognition. For example, a chemical entity may contain a number of long phrases and 
symbols. There are also a large number of hybrid entities that are partial, systematic and trivial.

A number of comprehensive chemical databases, such as PubChem [6], ChEBI [7] Jochem [8], ChemSpider [9], $\mathrm{MeSH}$ [10] and DrugBank [11] have been developed for various purposes, and could potentially be used as lexicons for chemical entity recognition. However, only a very limited number of chemical entity recognition systems have been developed and made publically available, probably due to the lack of large manually annotated corpora. The representative systems are Whatizit [12], OSCAR3/4 $[13,14]$ and ChemSpot [15]. Whatizit uses dictionary lookup to recognize chemical entities. OSCAR3/4 are machine learning-based systems that utilize maximum entropy models [16] on the OSCAR corpus. ChemSpot is a hybrid system that combines a machine learning-based classifier on SCAI corpus $[17,18]$ with a dictionary. Conditional random fields (CRF) [19] are used as the classifier in ChemSpot. So far, no comparative evaluation for different chemical entity recognition systems has been investigated on a standard corpus.

To accelerate the development of chemical entity recognition systems, The Spanish National Cancer Research Center (CNIO) and University of Navarra organized a challenge on Chemical and Drug Named Entity Recognition (CHEMDNER), as a part of BioCreative IV challenge (Track 2) [20-22]. The CHEMDNER challenge includes two individual subtasks: 1) Chemical Entity Mention recognition (CEM); and 2) Chemical Document Indexing (CDI). The subtask 1 is a typical named entity recognition task. The subtask 2 requires participants to rank chemical entities according to their importance in a chemical document. The challenge organizers provided manually annotated abstracts from PubMed $(10,000$ abstracts), of which 3,500 abstracts were used as a training set, 3,500 abstracts were used as a development set, and 3,000 abstracts were used as a test set.

In this paper, we described our systems for the CEM task. The systems first used a rule-based module for sentence boundary detection and tokenization, and then built machine learning classifiers based on CRF [19] and structured support vector machines (SSVM) [23] respectively. Both CRF and SSVM are state-of-the-art machine learning methods for NER, but SSVM has not been applied to chemical entity recognition yet. CRF is a discriminative undirected probabilistic graphical model, while SSVM is a discriminative model based on large margin theory. We also investigated the effects of three types of word representation (WR) features, generated by Brown clustering [24], random indexing [25] and skip-gram [26], on both the CRF-based and SSVM-based systems. WR is a new feature extraction technique that uses unsupervised learning algorithms to generate word-level features from an unlabeled corpus. Those WR features usually contain latent syntactic/semantic information of a word.

Our system was among the top ranked systems with an official micro F-measure of $85.05 \%$. After fixing a bug caused by inconsistent features, the performance was marginally improved with a micro F-measure of $85.20 \%$.

\section{Methods}

Figure 1 shows the architecture of our systems for the CEM subtask of CHEMDNER which consists of six components. Since the supplied raw chemical text was not well formatted, we developed rule-based modules to detect the boundary of sentences and tokenize them for each abstract at first module. We then realigned the preprocessed abstract back to the original one prior to the post-processing step of the pipeline. The other components of the systems are presented in the following sections in detail.

\section{Dataset}

The organizers collected 27,000 abstracts from the ISI Web of Knowledge relevant to various chemistry-related disciplines. 10,000 out of 27,000 abstracts were manually annotated with eight types of chemical entities based on a pre-defined guideline. The annotated abstracts were divided into three parts: a training set of 3,500 abstracts, a development set of 3,500 abstracts, and a test set of 3,000 abstracts. We used the training and development sets for system development, and evaluated our systems on the test set. The remaining 17,000 abstracts formed a test background set to avoid any manual correction of the predictions. Table 1 lists the counts of each type of entity in the training, development and test datasets.

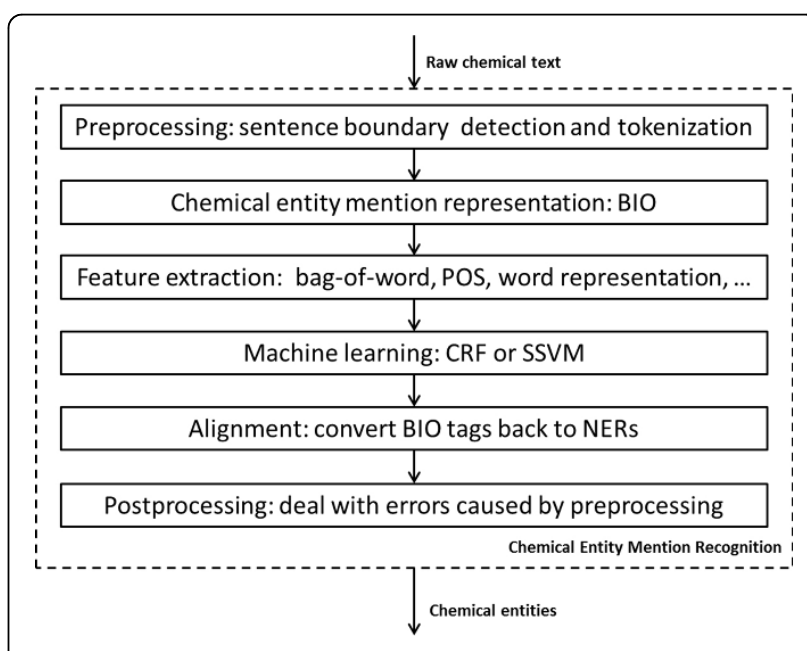

Figure 1 The architecture of our system for the CEM subtask. 
Table 1 Statistics of the dataset.

\begin{tabular}{lllll}
\hline Types & $\begin{array}{l}\text { Training } \\
\text { set }\end{array}$ & $\begin{array}{l}\text { Development } \\
\text { set }\end{array}$ & $\begin{array}{l}\text { Test } \\
\text { set }\end{array}$ & $\begin{array}{l}\text { Entire } \\
\text { corpus }\end{array}$ \\
\hline ABBREVIATION & 4,538 & 4,521 & 4,059 & 13,118 \\
\hline FAMILY & 4,090 & 4,223 & 3,622 & 11,935 \\
\hline FORMULA & 4,448 & 4,137 & 3,443 & 12,028 \\
\hline IDENTIFIER & 672 & 639 & 513 & 1,824 \\
\hline MULTIPLE & 202 & 188 & 199 & 589 \\
\hline SYSTEMATIC & 6,656 & 6,816 & 5,666 & 19,138 \\
\hline TRIVIAL & 8,832 & 8,970 & 7,808 & 25,610 \\
\hline NO CLASS & 40 & 32 & 41 & 113 \\
\hline ALL & 29,478 & 29,526 & 25,351 & 84,355 \\
\hline
\end{tabular}

\section{Chemical entity recognition}

In machine learning-based NER systems, the NER problem is usually converted into a classification problem by representing entities using specific tags. There are various representations for named entities [27] which are also suitable for chemical entities. In our study, we used BIO tags, a typical representation for named entities, to represent chemical entities, where " $\mathrm{B}$ ", "I" and "O" denote the beginning, inside and outside of an entity respectively. Therefore, the chemical entity recognition problem is converted into a classification problem wherein the task is to assign one of the three labels to each word. Figure 2 shows an example of the BIO representation, where the chemical entity "N-acetyl-L cysteine" is represented as "N/B -/I acetyl/I -/I L/I cysteine/I" after tokenization.

The features used in our systems include bag-of-word, orthographical information, morphological information, part-of-speech (POS), document structure information, domain knowledge and WR features. They are presented in detail as below:

- Bag-of-word: unigrams, bigrams and trigrams of tokens in window of $[-2,2]$.

- Orthographical information: word formation information, such as capital letters, numeric characters, and their combinations. All orthographic features used in our system are shown in Table 2.

- Morphological information: prefixes/suffixes of lengths from 2 to 5 and word shapes of tokens.

- POS: unigrams, bigrams and trigrams of POS (POS) in window of $[-2,2]$. Stanford tagger was used for POS tagging http://www-nlp.stanford.edu/software/tagger.shtml.

- Document structure information: is current token in a title or not?

Tokenized sentence: Thiol compounds such as $\mathbf{N}$ - acetyl - L cysteine BIO tag: Thiol/O compounds/O such/O as/O N/B -/I acetyl/I -/I L/I cysteine/I./O

Figure 2 An example of the BIO representation.
Table 2 Orthographic features used in our system.

\begin{tabular}{|c|c|c|c|}
\hline Feature & Regular Expression & Feature & Regular Expression \\
\hline ALLCAPS & $\wedge[A-Z]+\$$ & MANY_NUM & $\begin{array}{l}\wedge[0-9]\{1,2\}(,[0-9]\{1,2\}) \\
+\$\end{array}$ \\
\hline INITCAP & $\wedge[\mathrm{A}-\mathrm{Z}]^{*}$ & REAL_NUM & $\wedge-?[0-9]+\lfloor].[0-9]+\$$ \\
\hline HASCAP & $\wedge \cdot *[A-Z]{ }^{*} \$$ & INDASH & $\wedge([\backslash w+]\lfloor-]+)+\backslash w+\$$ \\
\hline SINGLECAP & $\wedge[A-Z] \$$ & HASDIGIT &.$^{*}[0-9]{ }^{*}$ \\
\hline PUNCTATION & $\wedge[, i: \backslash \backslash "] \$$ & IS_DASH & $\wedge[-]+\$$ \\
\hline INITDIGIT & $\wedge[0-9]^{*}$ & ROMAN & $\wedge[\mathrm{IVXDLCM}]+\$$ \\
\hline SINGLEDIGIT & $\wedge[0-9] \$$ & END_PUNC & $\wedge[?]] \$$ \\
\hline ALPHANUM & $\begin{array}{l}*^{*}[\mathrm{~A}-\mathrm{Z} \mathrm{Z}-\mathrm{Z}] .^{*}[0-9] .^{*} \\
.^{*}[0-9]{ }^{*}[\mathrm{~A}-\mathrm{Za}-\mathrm{Z}]{ }^{*}\end{array}$ & CAPSMIX & $\begin{array}{l}.^{*}[\mathrm{~A}-\mathrm{Z}] .^{*}[\mathrm{a}-\mathrm{Z}] .^{*} \\
\text {. }^{*}[\mathrm{a}-\mathrm{Z}] .^{*}[\mathrm{~A}-\mathrm{Z}]{ }^{*}\end{array}$ \\
\hline
\end{tabular}

- Domain knowledge: whether current token includes prefixes/suffixes of chemical compounds and drugs as shown in Table 3 chemical element list and drugs found in UMLS [28], cTAKES [29] and MetaMap [30].

- WR features: word representation features generated by Brown clustering [24], random indexing [25] and skip-gram [26].We followed the same method as in [31] to generate unsupervised word representation features using these three methods. For detailed information, please refer to [31].

We investigated two machine learning algorithms for chemical entity recognition: CRF and Structural Support Vector Machines (SSVM). CRF is a representative sequence labeling algorithm, which is a discriminative undirected probabilistic graphical model and is suitable for NER tasks. SSVM is a large margin-based discriminative algorithm for structural data, such as sequences, bipartite graph and trees. The SSVM combines the advantages of both CRF and SVM and is also suitable for sequence labeling problems such as NER tasks.

Furthermore, we defined some simple rules to fix a number of obvious errors as indicated below:

1) If an entity starts with the end of another one, combine them together to form a new entity.

2) If an entity only contains numbers and punctuations, remove it.

3) If there is unmatched ')' in the middle of an entity, combine it with the context from the previous '(' to the start of it to form a new entity.

4) If there is unmatched '(' in the middle of an entity, combine it with the context from the end of it to the next ')' to form a new entity.

\section{Experiments and evaluation}

In this study, we started with a baseline system that adopted features of bag-of-word, orthographic information, morphological information, POS, document structure 
Table 3 Prefixes/suffixes of chemical compounds and drugs.

\begin{tabular}{ll}
\hline prefixes & alk, meth, eth, prop, but, pent, hex, hept, oct, non, dec, undec, dodec, eifcos, di, tri, tetra, penta, hexa, hepta \\
\hline suffixes & ane, ene, yne, yl, ol, al, oic, one, ate, amine, amide \\
\hline
\end{tabular}

information and domain knowledge mentioned in the previous section. Then we evaluated the effects of three types of WR features: Brown clustering-based, random indexing-based and skip-gram-based word representations, by adding each of them individually to the baseline systems. Finally, we evaluated the performance of our systems when all three types of WR features were added. All WR features were derived from the entire unlabeled abstracts $(27,000)$ of the 2013 CHEMDNER challenge.

We used CRFsuite http://www.chokkan.org/software/ crfsuite/ and SVM ${ }^{\mathrm{hmm}}$ http://www.cs.cornell.edu/people/ tj/svm_light/svm_hmm.html as implementations of CRF and SSVM respectively. Both of them were trained on both the training and development sets, and their parameters were optimized on the development set when the models were trained on the training set.

The basic metrics used to evaluate system performance were micro precision, recall and F-measure as shown below:

$$
\begin{aligned}
& \text { precision }=\frac{\text { true positives }}{\text { true positives }+ \text { false positives }} \\
& \text { recall }=\frac{\text { true positive }}{\text { true positive }+ \text { false negetives }} \\
& F-\text { measure }=\frac{2 \times \text { precision } \times \text { recall }}{\text { precision }+ \text { recall }}
\end{aligned}
$$

where true positives corresponded to chemical entities correctly recognized, false positives corresponded to chemical entities wrongly recognized, and false negatives corresponded to chemical entities not recognized. All of them were calculated using the official evaluation tool provided by the organizers of the CHEMDNER challenge.

\section{Results}

Table 4 shows the performance of CRF-based and SSVM-based CEM classifiers on the test sets for subtask 1 of CHEMDNER, with the three WR features added either individually or together. The SSVM-based CEM system outperformed the CRF-based CEM system when using the same features. The difference F-measure between them ranged from $0.1 \%$ to $0.4 \%$. Addition of any type of the WR feature improved the performance of both the CRF-based and SSVM-based CEM systems. For example, when the skip-gram-based WR features were added to the baseline, the F-measure of the CRF-based
CEM system was improved by about $0.5 \%$ ( $84.54 \%$ vs 85.05\%), while the F-measure of the SSVM-based CEM system increased by about $0.2 \%$ ( $84.96 \%$ vs $85.20 \%$ ). Among the three types of WR features, the skip-grambased WR feature contributed to the maximal improvement as compared to the others when added to the baseline. When all the three types of WR features were added to the baseline, the F-measures of both the CRF-based and SSVM-based CEM systems were further improved, albeit only marginally. The highest F-measures achieved by the CRF-based and SSVM-based systems were $85.05 \%$ and $85.20 \%$ respectively.

\section{Discussion}

It is not unexpected that the SSVM-based CEM system outperformed the CRF-based CEM system in the current NER task. The same result has been obtained in several

studies on other NER tasks and shown to be due to the higher recall of the SSVM-based systems when same features are used [32-34]. Use of any of the WR features was beneficial to the chemical entity recognition task. When all the three types of WR features were added, both the CRF-based and SSVM-based systems showed better performance than the systems using only one type of WR features. The effects of the WR features to CEM are similar to that reported for biomedical named entity presented in an earlier study [31]. The improvement gains from WR features are mainly due to higher recalls, indicating that WR features can improve the generalization ability of machine learning-based CEM systems.

Table 4 The performance of CRF-based and SSVM-based CEM systems when different types of WR features were used.

\begin{tabular}{lllllll}
\hline Feature & \multicolumn{1}{l}{ CRF } & \multicolumn{5}{l}{ SSVM } \\
\cline { 2 - 7 } & Precision & Recall & $\begin{array}{l}\text { F- } \\
\text { measure }\end{array}$ & Precision & Recall & $\begin{array}{l}\text { F- } \\
\text { measure }\end{array}$ \\
\hline Baseline & 89.91 & 79.77 & 84.54 & 88.95 & 81.32 & 84.96 \\
\hline $\begin{array}{l}\text { Baseline }+ \\
\text { BC }\end{array}$ & 90.01 & 80.50 & 84.99 & 89.04 & 81.51 & 85.11 \\
\hline $\begin{array}{l}\text { Baseline }+ \\
\text { Rl }\end{array}$ & 89.52 & 80.60 & 84.83 & 88.43 & 82.03 & 85.12 \\
\hline $\begin{array}{l}\text { Baseline }+ \\
\text { SKIP }\end{array}$ & 89.86 & 80.71 & 85.04 & 88.88 & 81.73 & 85.15 \\
\hline All & 89.42 & 81.08 & 85.05 & 88.34 & 82.27 & 85.20 \\
\hline
\end{tabular}

* BC, RI and SKIP denote three different types of word representation features: Brown clustering-based, random indexing-based and skip-gram-based word representations respectively. 
The machine learning methods used in our systems were based on words (or tokens), which were generated by the preprocessing module shown in Figure 1. To assess the performance of machine learning methods more accurately, we studied the upper boundary performance of the machine learning-based system on the training set after preprocessing. We assigned BIO tags to all words in the training set and converted the words with tags back to chemical entities. We then compared these entities with the gold standard entities using the evaluation program and obtained the following micro precision, recall and F-measure: $97.79 \%, 97.84 \%$ and $97.82 \%$, respectively. It appears that the preprocessing module affects our system to a small extent (about $2.2 \%)$. However, a quick perusal of the difference in Fmeasure between our system and the best system of the CHEMDNER challenge ( $87.39 \%$ vs $85.20 \%$ ) [20] showed that it is comparable with the effect of preprocessing module. This observation has led us to determine that improvement of the preprocessing module would be a part of our future area of work or we could change our systems from word-based into char-based to avoid the performance loss caused by tokenization. Further, we also investigated the effect of the post processing module to our system. It improved the F-measure by about $0.2 \%$.

Compared with NER tasks in the newswire domain (F-measure about 90\%) [2], our reported performance on chemical entities was lower, indicating that CEM is more challenging and requires additional investigation and improvement. The possible directions of future pursuits include developing system ensemble approaches and utilizing specific patterns in the chemical domain for features.

\section{Conclusions}

In this study, we proposed a machine learning-based system for the CEM subtask of the CHEMDNER challenge, where CRF and SSVM were used as machine learning classifiers. We also investigated the effects of three types of WR features, generated by Brown clustering, random indexing and skip-gram, on both the CRF-based and SSVM-based systems. Our experiments on the CHEMDNER challenge corpus show that the SSVM-based systems outperformed the CRF-based systems when using the same features. Addition of any of the WR features was beneficial to the CEM task. Both the CRF-based and SSVM-based systems using all the three types of WR features showed better performance than the systems only using one type of the WR feature.

\section{Competing interests}

The authors declare that they have no competing interests.

\section{Authors' contributions}

The work presented here was carried out in collaboration between all. authors. BT, YF, XW and HX designed the methods and experiments. BT, YF YW, MJ and JW carried out the experiments. BT, YF, XW and HX analyzed the data, interpreted the results, and wrote the paper. All authors have attributed to, seen, and approved the manuscript.

\section{Acknowledgements}

This study is supported in part by grants: CPRIT (Cancer Prevention and Research Institute of Texas) R1307, NSFCs (National Natural Science Foundation of China) $(61402128,61173075$ and 61272383), Strategic Emerging Industry Development Special Funds of Shenzhen (ZDSY20120613125401420 and JCYJ20120613151940045)and CPSF (China Postdoctoral Science Funding) 2011M500669. We also thank the organizers of the CHEMDNER challenge.

\section{Declarations}

The publication fee of this paper is paid from JCYJ2012061315940045. This article has been published as part of Journal of Cheminformatics Volume 7 Supplement 1, 2015: Text mining for chemistry and the CHEMNDER track. The full contents of the supplement are available online at http://www. jcheminf.com/supplements/7/S1.

\section{Authors' details}

'Department of Computer Science, Harbin Institute of Technology Shenzhen Guraduate, Shenzhen, Guangdong, China. ${ }^{2}$ School of Biomedical Informatics, The University of Texas Health Science Center at Houston, Houston, Texas, USA. ${ }^{3}$ Department of Pharmacy, the First Affiliated Hospital, Harbin Medical University Harbin, Heilongjiang, China.

Published: 19 January 2015

\section{References}

1. Vazquez M, Krallinger M, Leitner F, Valencia A: Text Mining for Drugs and Chemical Compounds: Methods, Tools and Applications. Mol Informatics 2011, 30(6-7):506-519.

2. Tjong Kim Sang E, De Meulder F: Introduction to the CoNLL-2003 shared task: language-independent named entity recognition. presented at the Proceedings of the seventh conference on Natural language learning at HLT-NAACL 2003 2003, 4:142-147.

3. Kim J-D, Ohta T, Tsuruoka Y, Tateisi Y, Collier N: Introduction to the bioentity recognition task at JNLPBA. Proceedings of the International Joint Workshop on Natural Language Processing in Biomedicine and its Applications Stroudsburg, PA, USA; 2004, 70-75.

4. Smith L, Tanabe LK, nee Ando RJ, Kuo C-J, Chung I-F, Hsu C-N, Lin Y-S, Klinger R, Friedrich CM, Ganchev K, Torii M, Liu H, Haddow B, Struble CA, Povinelli RJ, Vlachos A, Baumgartner WA Jr, Hunter L, Carpenter B, Tsai RT-H, Dai H-J, Liu F, Chen Y, Sun C, Katrenko S, Adriaans P, Blaschke C, Torres R, Neves M, Nakov P, Divoli A, Maña-López M, Mata J, Wilbur WJ: Overview of BioCreative II gene mention recognition. Genome Biol 2008, 9(Suppl 2):S2.

5. Uzuner Ö, South BR, Shen S, DuVall SL: 2010 i2b2/NA challenge on concepts, assertions, and relations in clinical text. J Am Med Informatics Assoc JAMIA 2011, 18(5):552-556, Oct.

6. Wang Y, Xiao J, Suzek TO, Zhang J, Wang J, Bryant SH: PubChem: a public information system for analyzing bioactivities of small molecules. Nucleic Acids Res 2009, , 37 Web Server: W623-633, Jul.

7. Degtyarenko $K$, de Matos $P$, Ennis M, Hastings J, Zbinden M, McNaught A, Alcántara R, Darsow M, Guedj M, Ashburner M: ChEBl: a database and ontology for chemical entities of biological interest. Nucleic Acids Res 2008, , 36 Database: D344-350, Jan.

8. Hettne KM, Stierum RH, Schuemie MJ, Hendriksen PJM, Schijvenaars BJA, van Mulligen EM, Kleinjans J, Kors JA: A dictionary to identify small molecules and drugs in free text. Bioinforma Oxf Engl 2009, 25(22):2983-2991, Nov.

9. Pence HE, Williams A: ChemSpider: An Online Chemical Information Resource. J Chem Educ 2010, 87(11):1123-1124.

10. Van Camp AJ: The MeSH Vocabulary File and CHEMLINE. Med Ref Serv $Q$ 1984, 3(1):1-17.

11. Law V, Knox C, Djoumbou Y, Jewison T, Guo AC, Liu Y, Maciejewski A, Arndt D, Wilson M, Neveu V, Tang A, Gabriel G, Ly C, Adamjee S, Dame ZT, 
Han B, Zhou Y, Wishart DS: DrugBank 4.0: shedding new light on drug metabolism. Nucleic Acids Res 2014, , 42 Database: D1091-1097, Jan.

12. Rebholz-Schuhmann D, Arregui M, Gaudan S, Kirsch H, Jimeno A: Text processing through Web services: calling Whatizit. Bioinforma Oxf Engl 2008, 24(2):296-298, Jan.

13. Corbett $P$, Murray-Rust $P$ : High-Throughput Identification of Chemistry in Life Science Texts. Proceedings of the Second International Conference on Computational Life Sciences Berlin, Heidelberg; 2006, 107-118.

14. Jessop DM, Adams SE, Willighagen EL, Hawizy L, Murray-Rust P: OSCAR4: a flexible architecture for chemical text-mining. J Cheminformatics 2011, 3(1):41.

15. Rocktäschel T, Weidlich $M$, Leser U: ChemSpot: a hybrid system for chemical named entity recognition. Bioinforma Oxf Engl 2012, 28(12):1633-1640, Jun.

16. McCallum A, Freitag D, Pereira FCN: Maximum Entropy Markov Models for Information Extraction and Segmentation. Proceedings of the Seventeenth International Conference on Machine Learning, San Francisco, CA, USA 2000, 591-598.

17. Kolárik C, Klinger R, Friedrich CM, Hofmann-Apitius M, Fluck J: Chemical names: terminological resources and corpora annotation. Workshop on Building and evaluating resources for biomedical text mining (6th edition of the Language Resources and Evaluation Conference) 2008.

18. Klinger R, Kolárik C, Fluck J, Hofmann-Apitius M, Friedrich CM: Detection of IUPAC and IUPAC-like chemical names. Bioinforma Oxf Engl 2008, 24(13): i268-276, Jul.

19. Lafferty J, McCallum A, Pereira FCN: Conditional Random Fields: Probabilistic Models for Segmenting and Labeling Sequence Data. Dep Pap CIS 2001.

20. Krallinger M, Rabal O, Leitner F, Vazquez M, Oyarzabal J, Valencia A: Overview of the chemical compound and drug name recognition (CHEMDNER) task. Proceddings of the fourth BioCreative challenge evaluation workshop 2013, 2:2-33.

21. Krallinger $M$, Leitner F, Rabal O, Vazquez M, Oryazabal J, Valencia A: CHEMDNER: The drugs and chemical names extraction challenge. $J$ Cheminformatics 2014.

22. Krallinger M, Rabal O, Leitner F, Vazquez M, Salgado D, Lu Z, Leaman R, Lu Y, Ji D, Lowe DM, Sayle RA, Batista-Navarro RT, Rak R, Huber T, Rocktaschel T, Matos S, Campos D, Tang B, Xu H, Munkhdalai T, Ryu KH, Ramanan SV, Nathan S, Zitnik S, Bajec M, Weber L, Irmer M, Akhondi SA Kors JA, Xu S, An X, Sikdar UK, Ekbal A, Yoshioka M, Dieb TM, Choi M, Verspoor K, Khabsa M, Giles CL, Liu H, Ravikumar KE, Lamurias A, Couto FM, Dai H, Tsai RT, Ata C, Can T, Usie A, Alves R, Segura-Bedmar I, Martinez P, Oryzabal J, Valencia A: The CHEMDNER corpus of chemicals and drugs and its annotation principles. $J$ Cheminform 2015, 7(Suppl 1):S2.

23. Tsochantaridis I, Joachims $T$, Hofmann $T$, Altun $Y$ : Large margin methods for structured and interdependent output variables. J Mach Learn Res 2005, 6:1453-1484

24. Brown PF, deSouza PV, Mercer RL, Pietra VJD, Lai JC: Class-Based n-gram Models of Natural Language. Comput Linguist 1992, 18:467-479.

25. Kanerva P, Kristoferson J, Holst A: Random Indexing of Text Samples for Latent Semantic Analysis. Proceedings of the 22nd Annual Conference of the Cognitive Science Society 2000, 103-6.

26. Mikolov T, Chen K, Corrado G, Dean J: Efficient Estimation of Word Representations in Vector Space. CoRR 2013, abs/1301.3781.

27. Cho H-C, Okazaki N, Miwa M, Tsujii J: Named entity recognition with multiple segment representations. Inf Process Manag 2013, 49(4):954-965.

28. Unified Medical Language System (UMLS) - Home." [Online]. [http:// www.nlm.nih.gov/research/umls/, [Accessed: 02-Aug-2013].

29. Savova GK, Masanz JJ, Ogren PV, Zheng J, Sohn S, Kipper-Schuler KC, Chute CG: Mayo clinical Text Analysis and Knowledge Extraction System (cTAKES): architecture, component evaluation and applications. J Am Med Informatics Assoc JAMIA 2010, 17(5):507-513.

30. Aronson AR, Lang F-M: An overview of MetaMap: historical perspective and recent advances. J Am Med Informatics Assoc JAMIA 2010, 17(3):229-236, Jun

31. Tang B, Cao H, Wang X, Chen Q, Xu H: Evaluating Word Representation Features in Biomedical Named Entity Recognition Tasks. BioMed Res Int 2014, 2014, Mar

32. Tang $B$, Cao H, Wu Y, Jiang $M, X u$ H: Clinical entity recognition using structural support vector machines with rich features. Proceedings of the
ACM sixth international workshop on Data and text mining in biomedical informatics, New York, NY, USA 2012, 13-20.

33. Tang $B, C a o H, W u Y$, Jiang $M, X u H$ : Recognizing clinical entities in hospital discharge summaries using Structural Support Vector Machines with word representation features. BMC Med Inform Decis Mak 2013, 13(Suppl 1):S1, Apr.

34. Tang B, Wu Y, Jiang M, Chen Y, Denny JC, Xu H: A hybrid system for temporal information extraction from clinical text. $J$ Am Med Informatics Assoc JAMIA 2013, Apr.

doi:10.1186/1758-2946-7-S1-S8

Cite this article as: Tang et al:: A comparison of conditional random fields and structured support vector machines for chemical entity recognition in biomedical literature. Journal of Cheminformatics 20157 (Suppl 1):S8.

\section{Publish with ChemistryCentral and every scientist can read your work free of charge \\ "Open access provides opportunities to our colleagues in other parts of the globe, by allowing anyone to view the content free of charge." W. Jeffery Hurst, The Hershey Company. \\ - available free of charge to the entire scientific community - peer reviewed and published immediately upon acceptance \\ - cited in PubMed and archived on PubMed Central \\ - yours - you keep the copyright \\ Submit your manuscript here: \\ http://www.chemistrycentral.com/manuscript/<smiles>c1ccccc1</smiles> ChemistryCentral}

